\title{
Frequency of Intestinal Parasites Among Sudanese Patients with Irritable Bowel Syndrome
}

\author{
Sara Lavinia Brair ${ }^{1}$, Linda Bashier Eltayeb ${ }^{2}$, Sara Abdelghani Elgazy ${ }^{3}$, \\ Abdelsalam Mohammad Nail ${ }^{4}$, Alfatih Saifudinn Aljafari ${ }^{3,5, *}$ \\ ${ }^{1}$ Community Medicine Dep., Faculty of Medicine, Al-Neelain University, Khartoum, Sudan \\ ${ }^{2}$ Parasitology Dep., Faculty of Medical Laboratory Sciences, Omdurman Islamic University, Omdurman, Sudan \\ ${ }^{3}$ Parasitology Dep., Faculty of Medical Laboratory Sciences, Al-Neelain University, Khartoum, Sudan \\ ${ }^{4}$ Gastroenterology Dep., Faculty of Medicine, Omdurman Islamic University, Omdurman, Sudan \\ ${ }^{5}$ Pathology Dep., College of Medicine, Al Jouf University, Al Jouf, Saudi Arabia
}

\section{Email address:}

sara_lavinia@hotmail.com (S. L. Brair), lindarose009@hotmail.com (L. B. Eltayeb), saranaelgazy@gmail.com (S. A. Elgazy), abdelsalamnail@yahoo.com (A. M. Nail), aljafari@ju.edu.sa (A. S. Aljafari)

${ }^{*}$ Corresponding author

\section{To cite this article:}

Sara Lavinia Brair, Linda Bashier Eltayeb, Sara Abdelghani Elgazy, Abdelsalam Mohammad Nail, Alfatih Saifudinn Aljafari. Frequency of Intestinal Parasites Among Sudanese Patients with Irritable Bowel Syndrome. American Journal of Health Research.

Vol. 4, No. 4, 2016, pp. 82-85. doi: 10.11648/j.ajhr.20160404.12

Received: May 22, 2016; Accepted: June 3, 2016; Published: June 18, 2016

\begin{abstract}
The objective of this case control study is to identify the relationship between intestinal parasitic infection and Irritable Bowel Syndrome (IBS) among Sudanese patients. Method: Two hundred patients diagnosed with irritable bowel syndrome (IBS) and were attended Health Facilities at Khartoum State were enrolled, together with 99 apparently healthy controls. Patients were classified into three groups (IBS-all types, IBS with diarrhea (IBS-D), and IBS with constipation (IBSC)). Stool specimens were collected form study population and all were subjected to microscopic examination following wet saline preparation, formal-ether concentration technique, Z. N stain, and in vitro cultivation in Locke egg medium. Results: Intestinal parasites were detected in 32\% (64/200) of IBS-all types; and in 16.2\% (16/99) of control group (P. value 0.005). For patients, $20(10 \%)$ were positive for the Entamoeba cyst, $3(1.5 \%)$ Giardia lamblia cysts, and $41(20.5 \%)$ were positive for Blastocystis hominis cyst. For control, Entamoeba species were detected in $10(10.1 \%)$, G. lambelia one case (1.1\%), and $B$. hominis in $5(5.2 \%)$. Blastocystis hominis was significantly prevalent among IBS-all types than the control group $(P$. value $0.02)$. It is more frequent among patients with IBS- D than those with IBS-C (P. value 0.001$)$. Conclusion: This study concluded that, there is a statistical association between presence of intestinal parasites and IBS on study population. Blastocystis hominis was found more frequently detected parasite, and it was found significantly associated with IBS- diarrhea.
\end{abstract}

Keywords: Irritable Bowel Syndrome (IBS), Intestinal Parasites, Blastocystis hominis, Giardia Lamblia

\section{Introduction}

Irritable bowel syndrome (IBS) is a group of functional disorders that is associated with abdominal pain and alterations in bowel habit in the absence of an organic cause. The diagnosis IBS usually based on symptom criteria known as Rome criteria [1- 4]. It is one of the most commonly diagnosed gastrointestinal illnesses with prevalence rates of $10-15 \%$ in North America and Europe leading to an estimated cost to the
United States of 1.7 billion dollars in 2000. IBS is negatively affecting the quality of life of patients [5-7]. The pathophysiology of IBS is not well defined [8, 9]. Persistent low-grade inflammation may plays a role in IBS and it is one of proposed mechanism of IBS through persistent antigenic exposure as in persistent carriage. It is estimated that $7-31 \%$ of patients with infectious gastroenteritis go on to develop IBS (post- infectious IBS) [10, 11].

Intestinal parasitic infection is known to cause IBS-like symptoms that can be continuous, intermittent, and sporadic 
or recurrent [12]. Recent studies have described a possible role of protozoan parasites such as Blastocystis hominis, Dientamoeba fragilis, Giardia lamblia and Entamoeba histolytica in the etiology of IBS [2, 13].

The objective of this study is to identify the frequency of intestinal parasites among Sudanese patients with IBS.

\section{Material and Methods}

\subsection{Patients}

This is a case control study of a qualitative approach in which 200 patients with irritable bowel syndrome (IBS) were enrolled, together with 99 apparently healthy controls. Patients were selected by a professional gastroenterologist according to Rome III diagnostic criteria. Patients with, at least, one of the following conditions were excluded; Crohn's disease, ulcerative colitis, celiac sprue or colon cancer, within 12 months of the IBS diagnosis.

\subsection{Method}

Natural voided, fresh stool specimen was collected from each one of the study population. Specimens were subjected to; wet saline preparation, formal-ether concentration technique, Z. N stain, and in vitro cultivation.

For wet preparation, specimens were emulsified in normal saline on microscopic slide and then thoroughly examined under the microscope (X10 and X40 objectives). Formed specimens were stained with Lougl's Iodine for cyst identification. Parts of specimens (approximately 5 grams) were preserved in $20 \mathrm{ml}$ of SAF (sodium-acetate formalin) fixative for further permanent staining and formal ether concentration.

One gram (4 $\mathrm{ml}$ suspension) of each specimen was concentrated by Ritche formal-ether concentration technique which utilizes di-ethyl acetate instead of ether, sediment was then stained with Lougl's Iodine and was thoroughly examined under the microscope (X10 and X40 objectives) [12].

Part of SAF preserved specimen was smeared and prepared for staining by modified Ziehl- Neelsen stain. Following fixation in methanol for 3 minutes, smears were stained with Carbol fuchsin and decolorized in acid alcohol $(1 \% \mathrm{HCl}$ in methanol) and counter stained with $0.4 \%$ methylene blue [14].

Parasite in vitro cultivation:

Cultures were done by inoculating approximately $50 \mathrm{mg}$ of feces into Locke egg medium [15]. For cultivating $B$. hominis, starch free Locke egg medium was used. The cultures were incubated at $37^{\circ} \mathrm{C}$ and examined after 2-3 days. When vacuolar or granular forms of Blastocystis were observed, they were sub-cultured in fresh medium for another 3-4 days.

\subsection{Statistical Analysis}

Data was analyzed using IBM® Statistical Package for Social Science (SPSS) software. Frequencies and percentage were considered as qualitative measures. Significance of difference was analyzed by Chi-squared test, $\mathrm{P}$ value $<0.05$ was considered significant.

Ethical consideration:

This work received ethical clearance from the $\mathrm{FBC}$ of the College of Graduate, Al-Neelain University. Informed consent was obtained from each participant.

\section{Results}

The study group was composed of 200 IBS patients (119 females and 81 males) with a mean age of $37.1( \pm 1.6)$ years. The demographics of the study population are summarized in Table-1.

Patients were classified into three groups; IBS all types (having IBS regardless the type); the second group contains patients with IBS- diarrhea (IBS-D); and the third group contains patients with constipation predominant IBS (IBS-C).

Intestinal parasites were detected in $32 \%(64 / 200)$ of IBSall types; and in $16.2 \%(16 / 99)$ of control group. There was statistical difference between IBS-all types and control group in this regard (P. value 0.005). Examination of patients' stool by the different methods collectively revealed that $20(10 \%)$ were positive for the Entamoeba cyst, 3 (1.5\%) G. lamblia cysts, and $41(20.5 \%)$ were positive for $B$. hominis cyst. For control group, Entamoeba species were detected in 10 (10.1\%), Giardia lambelia 1 (1.1\%), and Blastocystis hominis in $5(5.2 \%)$. Blastocystis hominis was more prevalent among IBS-all types than the control group (P. value 0.02$)$. Table-2 illustrates the frequency of intestinal parasites among study population.

Blastocystis hominis was more prevalent among patients with IBS-D than those with IBS-C (P. value 0.000). $B$. hominis was detected in $12.8 \%$ of patients with IBS-D, and in of patients with IBS-C. $89.1 \%$ of $B$. hominis were detected among IBS-all type patients. Table-3 illustrates the detailed frequencies and percentage of intestinal parasites among IBS major subtypes.

Table 1. Frequency of Age among Irritable bowel syndrome and control Group.

\begin{tabular}{lll}
\hline Age group & Patients & Control \\
\hline $20-35$ & $55(27.5 \%)$ & $46(46.5 \%)$ \\
$36-50$ & $81(40.5 \%)$ & $43(43.4 \%)$ \\
$51-75$ & $64(32.0 \%)$ & $10(10.1 \%)$ \\
Total & 200 & 99 \\
\hline
\end{tabular}

Table 2. Frequency and percentage of intestinal parasites among patients (IBS-all types) and control.

\begin{tabular}{lll}
\hline & Patients (N\%) & Control (N\%) \\
\hline Entamoeba* & $20(10 \%)$ & $10(10.1 \%)$ \\
Giardia lamblia & $3(1.5 \%)$ & $1(1 \%)$ \\
B. hominis & $41(20.5 \%)$ & $5(5.1 \%)$ \\
Total parasites & $46(32 \%)$ & $16(16.2 \%)$ \\
Negative & $136(68 \%)$ & $83(83.8 \%)$ \\
Total & 200 & 99 \\
$P$ value 0.005 & & \\
\hline
\end{tabular}

* (E. histolytica, E. coli, I. buetchelli, and E. nana) 
Table 3. Frequency and percentage of intestinal parasites among patients with IBS- $D$ and IBS-C.

\begin{tabular}{llll}
\hline Parasites & IBS-D (120) & IBS-C (103) & P. value \\
\hline Entamoeba species & $15(12.5 \%)$ & $5(4.85 \%)$ & 0.65 \\
Giardia lambelia & $2(1.66 \%)$ & 0 & 0.5 \\
B. hominis & $23(19.16 \%)$ & $2(1.94 \%)$ & 0.000 \\
Total & $40(33.33 \%)$ & $7(6.8 \%)$ & \\
\hline
\end{tabular}

* (E. histolytica, E. coli, I. buetchelli, and E. nana)

\section{Discussion}

Association between intestinal parasite infection and IBS is a matter of concern; at least, it is not excluded. Findings of this study go on the stream with many similar works [16]. Several studies have implicated intestinal protozoa in the differential diagnosis of IBS because they cause symptoms resembling IBS or may cause significant flares of IBS with acquisition [17]. Furthermore, they may lead to IBS, secondary to ongoing low grade inflammation through persistent immune activation as a result of antigenic exposure as in persistent carriage/infection that frequently occurs in intestinal parasitic diseases [1].

Intestinal protozoa identified in this study included: $B$. hominis, G. lamblia, and Entamoeba species. Several studies have implicated these parasites in the etiology of IBS $[2,18$ 20]. However, fewer studies found no association between exposure to those parasites and predisposition to IBS symptoms [21]. In our case, B. hominis was the most prevalent detected protozoa as it was detected in $41(20 \%)$ of IBS patients and in $5(5.1 \%)$ of control. It was lesser than the findings of Yakoob et. al., who reported that fecal carriage of B. hominis occurs more frequently in IBS patients (46\%) than in the control group (7\%) [22]. Symptoms that attributed to infection with $B$. hominis are non-specific, however, they include diarrhea, abdominal pain, cramps or discomfort and nausea, which mimic the IBS-like symptoms [23]. Other augmenting finding of this study is that, B. hominis was found significantly associated with IBS-D (diarrhea) subtype. G. lamblia infection also may mimic IBS. Evidence for postinfectious IBS secondary to low-grade inflammation was demonstrated by D'Anchino et. al., [19]. In the present study, G. lamblia cyst was detected in three (1.5\%) of IBS patients; this was similar to the results of Grazioli et. al., [24].

As a long-term infection, a correct diagnosis and treatment should be given for Giardia lamblia and E. histolytic [12]. However, they may be innocent bystanders, as occurs with asymptomatic carriage or infections by nonpathogenic protozoa. In this study, stool microscopy revealed protozoal cysts in a significant number of IBS patients $(32 \%)$ compared to controls $(16.2 \%)$. This result is consistent with Bujanda et. al., who showed that, $49 \%$ of the patients with symptoms suggestive of gastrointestinal functional disorders were, or had previously been, diagnosed with parasitic infection [19].

Parasites of Genus Entameba including E. histolytica was detected in twenty patient (10\%) and $10(10.1 \%)$. Unless there is acute dysentery, the role of Entamoeba carriage is not significant for IBS irritation. Early studies implicated amoebic dysentery in the development of IBS among British soldiers returning from Egypt. On the contrary, several other studies from India have suggested that exposure to E. histolytica did not predispose patients to IBS symptoms [24, 25].

The findings of this study together with many others prove that, there is a relationship between IBS and intestinal parasites. This relationship differs according to the Genus of parasite and the type of IBS. There is a consensus, that $B$. hominis is associated with IBS-D, and the association of $G$. lambelia is variable and may be depend on cyst shedding. Entamoeba is likely associated with post inflammatory-IBS. It still not clear whether the parasites cause IBS, or just provoke some tissue reactions that exacerbate already existing disorder. We can point to inflammatory cytokines, mast cells activity, or neurotransmitter accumulation in the tissue milieu. We are investigating this issue meanwhile.

\section{Conclusion and Recommendation}

This study concluded that, there is a statistical association between presence of intestinal parasites and IBS on study population. Blastocystis hominis was found more frequent detected parasite, and it is significantly associated with IBSdiarrhea.

\section{Acknowledgement}

We would like to thank all participants in this study. Our thanks extended to the department of Medical Parasitology at the Faculty of Medical Laboratory Sciences, Al-Neelain University for their kind assistance and help.

\section{References}

[1] Sinha P, Ghoshal UC, Choudhuri G, Naik S, Ayyagari A, Naik SR. Does Entamoeba histolytica cause irritable bowel syndrome? Indian J Gastroenterol 1997; 16 (4): 130- 133.

[2] Wong KH, Ng GC, Lin RT, Yoshikawa H, Taylor MB, Tan KS. Predominance of subtype 3 among Blastocystis isolates from a major hospital in Singapore. Parasitol Res 2008; 102 (4): 663-670.

[3] Ramirez-Miranda ME1, Hernandez-Castellanos R, LopezEscamilla E, Moncada D, Rodriguez-Magallan A, PagazaMelero C, Gonzalez-Angulo A, Flisser A, Kawa- Karasik S, Maravilla P. Parasites in Mexican patients with irritable Bowel syndrome: a case-control study. Parasites Vectors 2010; 3: 96.

[4] Tan KSW. New insights on classification, identification and clinical relevance of Blastocystis spp. Clin Microbiol Rev 2008; 21 (4): 639-665.

[5] Occhipinti K, Smith JW. Irritable Bowel Syndrome: A Review and Update. Clinics in Colon and Rectal Surgery. 2012; 25 (1): 46-52. 
[6] Eltayeb LB, Aljafari AS, Brair LS, Algazy SA, Nail AM. Assessment and Evaluation Of The Effect Of Irritable Bowel Syndrome (IBS) On The Health Related Quality Of Life Among Sudanese Patients. Am. J. Life. Sci. Res 2015; 3 (4): 254-459.

[7] OKeke EN, Agaba EI, Gwamzhi L, Achinge GI, Malu AO. Prevalence of irritable bowel syndrome in a Nigerian student population. Afr. J. Med. Med. Sci 2005; 34: 33-36

[8] Thompson WG, Heaton KW, Smyth GT, Smyth C. Irritable bowel syndrome in general practice: Prevalence, characteristics and referral. Gut. 2000; 46: 78-82.

[9] Levy RL, Jones KR, Whithead WE, Feld SI, Talley NJ, Corey LA. Irritable bowel syndrome in twins: heredity and social learning both contribute to etiology. Gastroenterology 2001; 121: 799-804.

[10] Wang J, Lu S, Zhao S. Post-infectious and non post-infectious irritable bowel syndrome: A comparative study. Pakistan Journal of Medical Sciences. 2016; 32 (1): 116-119. doi: 10.12669/pjms.321.8628.

[11] Rodriguez LA, Ruigomez A. Increased risk of irritable bowel syndrome after bacterial gastroenteritis: cohort study. BMJ $1999 ; 318,565-566$.

[12] Stark D, vanHal S, Marriott D, Ellis J, Harkness J. Irritable bowel syndrome: a review on the role of intestinal protozoa and the importance of their detection and diagnosis. Int $\mathrm{J}$ Parasitol 2007 Jan; 37 (1): 11-20.

[13] Ragavan ND, Kumar S, Chye TT, Mahadeva S, Shiaw-Hooi H. Blastocystis sp. in Irritable Bowel Syndrome (IBS)-Detection in Stool Aspirates during Colonoscopy. PLoS One. 2015 Sep 16; 10 (9): e0121173. doi: 10.1371/journal. pone.0121173. eCollection 2015.

[14] Clarke SC, McIntyre M. Modified detergent Ziehl-Neelsen technique for the staining of Cyclospora cayetanensis. J Clin Pathol 1996; 49 (6): 511-512.

[15] Clark CG, Diamond LS. Methods for cultivation of luminal parasitic protists of clinical importance. Clin Microbiol. Rev 2002 Jul; 15 (3): 329-334.

[16] Longstreth GF, Thompson WG, Chey WD, Houghton LA,
Mearin F, Spiller RC. Functional bowel disorders. Gastroenterology 2006; 130 (5): 1480-1491.

[17] Hussain R, Jaferi W, Zuberi S, Baqai R, Abrar N, Ahmed A, Zaman V. Significantly increased IgG2 subclass antibody levels to Blastocystis hominis in patients with irritable bowel syndrome. Am J Trop Med Hyg 1997; 56 (3): 301-306.

[18] Eltayeb LB, Brair SL, Aljafari AS. The impact of intestinal protozoan parasites among Irritable Bowel Syndrome patients in Khartoum state. Al Neelain Medical Journal 2013; 3 (9): 47-57.

[19] Bujanda L, Gutierrez Stampa MA, Caballeros CH, et al. Trastornos gastrointestinales en Guatemala y su relacion con infecciones parasitarias [Gastrointestinal disorders in Guatemala and their relationship with parasitic infections]. Ann Med Interne 2002; 19 (1): 36.

[20] D'Anchino M, Orlando D, De Feudis L. Giardia lamblia infections become clinically evident by eliciting symptoms of irritable bowel syndrome. J Infect 2002; 45 (3): 169-172.

[21] Stark D1, Beebe N, Marriott D, Ellis J, Harkness J. Prospective study of the prevalence, genotyping and clinical relevance of Dientamoeba fragilis infections in an Australian population. J Clin Microbiol 2005; 43 (6): 2718-2723.

[22] Yakoob J, Jafri W, Jafri N, Khan R, Islam M, Beg MA, Zaman V. Irritable bowel syndrome: in search of an etiology: role of Blastocystis hominis. Am J Trop Med Hyg 2004; 70 (4): 383385 .

[23] Tungtrongchitr A, Manatsathit S, Kositchaiwat C, Ongrotchanakun J, Munkong N, Chinabutr P, Leelakusolvong S, Chaicumpa W. Blastocystis hominis infection in irritable bowel syndrome patients. Southeast Asian J Trop Med Public Health 2004; 35 (3): 705-710.

[24] Grazioli B1, Matera G, Laratta C, Schipani G, Guarnieri G, Spiniello E, Imeneo M, Amorosi A, Foca A, Luzza F. Giardia lamblia infection in patients with irritable bowel syndrome and dyspepsia: a prospective study. World J Gastroenterol 2006; 12 (12): 1941-1944.

[25] Corcoran GD, O'Connell B, Gilleece A, Mulvihill TE. Entamoeba coli as possible cause of diarrhea. Lancet 1991; 338 (8761): 254. 